\title{
Mir-421 in plasma as a potential diagnostic biomarker for precancerous gastric lesions and early gastric cancer
}

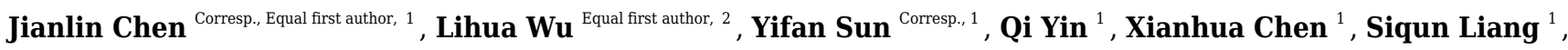 \\ Qingyan Meng ${ }^{1}$, Haihua Long ${ }^{2}$, Fangying $\mathrm{Li}^{2}{ }^{2}$, Changjun Luo ${ }^{3}$, Xiaorong Xiao ${ }^{4}$ \\ ${ }^{1}$ Department of Clinical Laboratory, Affiliated Liutie Central Hospital of Guangxi Medical University, Liuzhou, Guangxi, China \\ 2 Department of Digestive internal medicine, Affiliated Liutie Central Hospital of Guangxi Medical University, Liuzhou, Guangxi, China \\ 3 Department of Internal Medicine-Cardiovascular, Affiliated Liutie Central Hospital of Guangxi Medical University, Liuzhou, Guangxi, China \\ 4 Department of Science and Education, Affiliated Liutie Central Hospital of Guangxi Medical University, Liuzhou, Guangxi, China \\ Corresponding Authors: Jianlin Chen, Yifan Sun \\ Email address: cjl801900@163.com, sunyifan13@126.com
}

Objective. MicroRNA (miR)-421 plays a key role in cancer progression. It has been reported that circulating miR-421may be a potential tumor marker for the diagnosis of several cancers. However, the role of miR-421 in plasma as a potential biomarker in the diagnosis of precancerous gastric lesions (Pre) and early-stage gastric cancer (GC) remains poorly understood. In this study, we investigated miR-421 in plasma as a novel potential biomarker for the detection of precancerous gastric lesions and early-stage (GC).

Materials \& Methods. The miRNA content was determined by quantitative real-time polymerase chain reaction (qRT-PCR). MiR-421 content in all subjects was normalized by endogenous miRNA (miR-16). The diagnostic value of miR-421 for Pre and GC was assessed by comparing receiver operating characteristic $(R O C)$ analysis with traditional tumor markers, including CEA, CA125, CA153, CA211 and CA50. The correlation between the expression of miR-421 and the pathological characteristics of Pre and GC was analyzed. Results. Elevated expression of miR-421 in plasma can robustly distinguish the normal population from Pre and GC cases, especially in the early stages of gastric cancer cases (all $p<0.05$ ). The ROC analyses showed that the area under the ROC curve (AUC), sensitivity, accuracy and Youden index of miR-421 were superior to traditional tumor markers (CEA, CA125, CA153, CA211, and CA50) in GC diagnosis, while its specificity was higher than CEA, CA153 and CA50 (all $p<0.05$ ). MiR-421 in plasma had higher AUC value than AFP, CA153, CA211 and CA50 in the diagnosis of Pre (all $p<0.05$ ), while specificity, accuracy and Youden index of miR-421 was only lower than CA211. The efficiency of miR-421 in the diagnosis of GC was significantly higher than that of CA211 and CA50, and it was significantly higher than CA153, CA211 and CA50 in the diagnosis of Pre (all $p<0.05)$. In addition, up-regulation of miR-421 occurred initially in precancerous gastric lesions as well as in the early stage of GC. Conclusions. Overexpression of plasma Peer] reviewing PDF | (2018:10:31786:2:0:NEW16 Apr 2019) 
miR-421 is a novel biomarker for the detection of precancerous lesions and early gastric cancer. 
1 Mir-421 in plasma as a potential diagnostic biomarker for

\section{2 precancerous gastric lesions and early gastric cancer}

3 Jianlin Chen ${ }^{1, *}$, Lihua $\mathrm{Wu}^{2}$, Yifan $\mathrm{Sun}^{3}$, Qi Yin ${ }^{4}$, Xianhua Chen ${ }^{5}$, Siqun Liang ${ }^{6}$, Qingyan Meng ,

4 Haihua Long ${ }^{8}$, Fangying $\mathrm{Li}^{9}$, Changjun $\mathrm{Luo}^{10}$, Xiaorong Xiao ${ }^{11}$

5 Department of Clinical Laboratory ${ }^{1,3,4,5,6,7}$, Department of Digestive internal medicine ${ }^{2,8,9}$,

6 Department of Internal Medicine-Cardiovascular ${ }^{10}, \mathrm{~d}$ Department of Science and Education ${ }^{11}$,

7 Affiliated Liutie Central Hospital of Guangxi Medical University, Liuzhou, Guangxi, China

8 Corresponding Author: Jianlin Chen, Yifan Sun

914 Fei-e Road, Liuzhou, Guangxi, 545007, China

10 Email address: cj1801900@163.com; sunyifan13@126.com

11 ABSTRACT

Objective. MicroRNA (miR)-421 plays a key role in cancer progression. It has been reported that circulating miR-421may be a potential tumor marker for the diagnosis of several cancers. However, the role of miR-421 in plasma as a potential biomarker in the diagnosis of precancerous gastric lesions (Pre) and early-stage gastric cancer (GC) remains poorly understood. In this study, we investigated miR-421 in plasma as a novel potential biomarker for the detection of precancerous gastric lesions and early-stage (GC).

Materials \& Methods. The miRNA content was determined by quantitative real-time polymerase chain reaction (qRT-PCR). MiR-421 content in all subjects was normalized by endogenous miRNA (miR-16). The diagnostic value of miR-421 for Pre and GC was assessed by comparing receiver operating characteristic (ROC) analysis with traditional tumor markers, including CEA, CA125, CA153, CA211 and CA50. The correlation between the expression of miR-421 and the pathological characteristics of Pre and GC was analyzed.

Results. Elevated expression of miR-421 in plasma can robustly distinguish the normal population from Pre and GC cases, especially in the early stages of gastric cancer cases (all $p$ $<0.05$ ). The ROC analyses showed that the area under the ROC curve (AUC), sensitivity, accuracy and Youden index of miR-421 were superior to traditional tumor markers (CEA, CA125, CA153, CA211, and CA50) in GC diagnosis, while its specificity was higher than CEA, CA153 and CA50 (all $p<0.05$ ). MiR-421 in plasma had higher AUC value than AFP, CA153, 
30 CA211 and CA50 in the diagnosis of Pre (all $p<0.05)$, while specificity, accuracy and Youden index of miR-421 was only lower than CA211. The efficiency of miR-421 in the diagnosis of GC was significantly higher than that of CA211 and CA50, and it was significantly higher than CA153, CA211 and CA50 in the diagnosis of Pre (all $p<0.05$ ). In addition, up-regulation of miR421 occurred initially in precancerous gastric lesions as well as in the early stage of GC.

Conclusions. Overexpression of plasma miR-421 is a novel biomarker for the detection of precancerous lesions and early gastric cancer.

\section{INTRODUCTION}

Gastric cancer (GC) is one of the most common malignant tumors in China(Zheng et al. 2019). Due to the lack of tumor markers and specific symptoms and signs, most tumors have metastasized by the time of diagnosis. Studies have shown that patients with advanced GC have a poor prognosis with a 5 -year survival rate of less than $25 \%$ (Chan et al. 2001). However, if intervention and treatment are performed in the pre- or early stages of gastric cancer, the fiveyear survival rate will rise to $95 \%$ (Craanen et al. 1991). Therefore, it is very important to detect and treat gastric cancer at an early stage.

Gastric cancer originates from the malignant and aggressive proliferation of gastric mucosal cells. Most are developed and evolved by the chronic inflammation of gastric mucosa under the influence of multiple factors, mainly in four stages (chronic superficial gastritis $\rightarrow$ chronic atrophic gastritis $\rightarrow$ intestinal metaplasia and dysplasia $\rightarrow$ gastric cancer). Chronic atrophic gastritis is currently recognized as a precancerous disease, intestinal metaplasia and dysplasia is a precancerous lesion closely related to the development of gastric cancer. Currently, Gastroscopy combined with biopsy is the gold standard for diagnosis of gastric cancer. However, it may not be appropriate to carry out large-scale screening and early detection in most countries including China due to acceptability, availability, cost and other reasons. GC related tumor markers such as, cancer embryo antigen (CEA), pepsinogen (PG), carbohydrate antigen 199(CA199) and carbohydrate antigen 724(CA724), gastrin-17(G17) have been used clinically for many years, but many do not have sufficient sensitivity and specificity for GC screening(He et al. 2013; Pectasides et al. 1997; Yang et al. 2014). Studies on their role in monitoring early gastric cancer have rarely been reported. Therefore, there is a need for reliable and non-invasive biomarkers for early detection and mass screening of GC. 
62 MicroRNAs (miRNAs) are a class of endogenous non-coding RNA molecules (length of 18-25

63 nucleotides) that exert their roles by base pairing between the seed region of miRNA or 3'-un-

64 translated regions (3'-UTR) of the target gene(Lee \& Ambros 2001). Studies have confirmed that 65 miRNAs' expression are frequently de-regulated in human tumors(Hernando 2007; Lu et al.

66 2005). Recently, circulating miRNAs (miRNAs present in blood) have attracted attention due to their stability and ease of measurement. Many circulating miRNAs have been quantified and identified by qRT-PCR as tumor markers to detect GC, such as miR-223, miR-21, miR-218(Li et al. 2012; Zhou et al. 2015), and miR-18a(Tsujiura et al. 2015) in plasma, and miR-20a(Yang et diagnosis of precancerous gastric lesions and early-stage gastric cancer, and most of these studies have not compared the value of these miRNAs with traditional tumor markers in the diagnosis of gastric cancer and precancerous lesions.

MicroRNA-421 (miR-421) is a molecule that has been studied extensively. It has been investigated and found to be expressed aberrantly in various types of cancer(Li et al. 2018; Wang et al. 2018; Zhou et al. 2016). Previous studies showed that miR-421 rises notably in tissue and gastric juice, as well as in blood mononuclear cells and serum of gastric cancer(Liu et al. 2015; Zhang et al. 2012a; Zhao et al. 2015), suggesting that miR-421 seems to be an ideal biomarker for gastric cancer. However, most of these studies failed to point out at which stage in the evolution of gastric cancer miR-421 can be detected in the peripheral blood, and an optimal circulating miRNA should have the ability to distinguish different phases in the course of cancer development. Some critical issues remain unresolved. Firstly, the expression of miR-421 in the plasma of precancerous patients has not been fully investigated. More importantly, the value and significance of miR-421 in the early diagnosis of gastric cancer are still not clearly stated. The present study aimed to clarify the diagnostic value of miR-421 in the early stage of gastric cancer and in the precancerous lesions of gastric cancer and to explore its relation with the progression of GC.

\section{Materials \& methods}

\section{Clinical specimens}

Plasma samples from 90 GC patients, 89 patients with precancerous (Pre) lesions and 45 normal healthy controls (NC) were collected at Affiliated Liutie Central Hospital of Guangxi Medical University in Guangxi province, China and the levels of miR-421 were screened by qRT-PCR. GC patients were confirmed by gastroscopy combined with biopsy. Tumor type and stage were 
identified according to the Union of International Cancer Control (UICC) tumor-node-metastasis (TNM) system, 7th edition. The histology of all patients was evaluated according to the World Health Organization (WHO) criteria. Patients who had received Radiation therapy or chemotherapy were excluded from the study. The pathological outcomes of GC and Pre patients were obtained by two experienced pathologists. In addition, 45 healthy people without a previous history of diabetes, heart disease, hypertension, and cancer were chosen as normal controls (NC). We compared the plasma levels of miR-421 in patients with gastric cancer, and precancerous lesions, and in normal controls to assess the feasibility of miR-421 as a novel non-invasive biomarker for early GC detection. The expression levels of conventional tumor markers in GC, Pre, and normal controls were analyzed to evaluate the specificity and sensitivity of miR-421 for early diagnosis of gastric cancer. This study was subject to approval by the Ethics Committee of the Affiliated Liutie Central Hospital of Guangxi Medical University and written informed consent was issued by all study participants.

\section{Plasma preparation and storage}

$8 \sim 10 \mathrm{ml}$ of venous blood of each participant was collected using ethylenediaminetetraacetic acid (EDTA) anticoagulative tubes immediately. Cell-free plasma was separated within $2 \mathrm{~h}$ after collection by $3000 \mathrm{rpm}, 5 \mathrm{~min}$ centrifugation to prevent contamination by cellular nucleic acids. Plasma transferred into RNase/DNase-free tubes was stored at $-80^{\circ} \mathrm{C}$ until MicroRNA extraction. Plasma samples for conventional tumor markers and Helicobacter pylori antibody determination were separated and kept at $-20^{\circ} \mathrm{C}$ until assayed.

\section{MicroRNA extraction and qRT-PCR assays}

MicroRNAs were isolated from 200 $\mu$ l plasma utilizing Blood (Serum/Plasma) MicroRNA

Extraction and Purification Kit (spin column) (Novland Co., Ltd, Shanghai, China) following the manufacturer's protocol. The concentrations of miRNA from plasma samples were quantified using a NanoQ Micro-volume Spectrophotometer, (CapitalBio, Beijing, China). The initial template for qRT-PCR was 2ul. Circulating miRNAs expression was determined using one step Stemaim-it miR qRT-PCR Kit Quantitation (Taqman probes) (Novland Co., Ltd, Shanghai, China), while miR-16 served as a reference miRNA. Expression levels of target miRNAs were performed on the ABI-7500 PCR system and calculated by the cycle threshold $(\mathrm{Ct})$ values with 
124 SDS 2.0 software (Applied Biosystems, Foster City, CA, USA). Samples with Ct values greater 125 than 30 were excluded. The levels of miR-421 in different samples were calculated by the $2^{-\Delta \mathrm{Ct}}$ 126 method, in which $\Delta \mathrm{Ct}=\mathrm{Ct}(\mathrm{miR}-421)-\mathrm{Ct}(\mathrm{miR}-16)$.

127

\section{Conventional tumor markers and Helicobacter pylori antibodies}

Conventional tumor markers were tested by electrochemiluminescence immunoassay according to the standard procedure of Roche Company's kit and Roche E170 automatic immunity analyzer. Helicobacter pylori antibodies were assayed following the Anti-helicobacter pylori antibody detection kit (ELISA) (Beier, biological engineering co. Ltd, Beijing, China).

\section{Statistical analysis}

All data were analyzed using MedCalc statistical software (v18.2.1) and GraphPad Prism 7.0. The levels of miR-421 and conventional tumor markers in plasma among Pre, GC patients, and health cases were established by the Mann Whitney test. Receiver operating characteristic curves (ROC) and the AUC were constructed to evaluate the diagnostic values of each tumor markers. The optimal cut-off thresholds were determined by using the highest Youden index. The Chisquared test was utilized to analyze the associations between the expression level of miR-421 and the clinicopathological factors. All differences were examined statistically significant at $p<$ 0.05 .

\section{Results}

\section{Expression of miR-421 and tumor markers in plasma of GC patients, Pre patients,} and normal controls

This study recruited 90 GC patients, 89 Pre patients, and 45 normal healthy subjects as controls. Plasma miR-421 levels were detected by qRT-PCR and analyzed using miR-16 as a reference miRNA. To verify whether miR-16 was adaptable to be a reference miRNA in our system, the expression levels of miR-16 were detected. As shown in Fig. 1A, there was no statistical difference in the expression of miR-16 between the normal and gastric cancer group, or the normal and precancerous group, which implied that miR-16 was a suitable reference control for detecting miR-421 in plasma samples. The qRT-PCR results showed that miR-421 was significantly up-regulated in GC and Pre patients compared with the control group (all $p<0.001$, Figs. 1B,1C). The concentrations of CEA, CA125, CA153, CA211, and CA50 in the GC group 
153 were significantly higher than in the healthy controls (all $p<0.05$, Table 1 ). Moreover, the concentration of CA211 and CA50 in the Pre group was significantly higher than the healthy controls (all $p<0.05$, Table 1). Of note, compared with the precancerous group, the concentration of AFP and CA153 was significantly higher in the normal group (all $p<0.05$, Table 1 ). miR-421 in plasma had higher diagnostic value as a non-invasive biomarker than traditional tumor markers for gastric cancer

To further evaluate the diagnostic value of miR-421 for GC, receiver-operating characteristic (ROC) curve analyses were performed (Fig. 2A and Table 2). According to the ROC curve, when the cut-off of 3.23 , the optimal sensitivity and specificity of miR-421 were $96.67 \%$ and $95.56 \%$ respectively $[\mathrm{AUC}=0.981(0.942-0.997)]$. To further clarify the diagnostic efficiency of miR-421 in gastric cancer, the diagnostic values of traditional tumor markers were compared to those of miR-421. Fig. 2B shows that the AUC, sensitivity, accuracy and Youden index of miR-421 were higher than all five conventional biomarkers, while the specificity was higher than CEA, CA153, and CA50. Table 3 shows the direct comparison of the ROC curves of circulating miR-421 with five conventional tumor markers. The diagnostic efficacy of miR-421 was significantly higher than CEA, CA153, CA211, and CA50 (all $p<0.05$ ). In order to determine whether the plasma level of miR-421 has clinical value for GC diagnosis, the false positive rate (FPR) and false negative rate (FNR) and diagnosis efficiency were calculated. As shown in Table 2, the results indicate that the FNR of miR-421 was lower than all five conventional biomarkers, while the FPR was lower than CEA, CA153, and CA50, which indicated that miR421 has a high diagnostic value for GC. These results indicate that the plasma miR-421 has greater diagnostic value for GC than five conventional tumor markers and can be used as a noninvasive diagnostic marker for gastric cancer.

\section{miR-421 in plasma could be used to distinguish early-stage GC patients from} healthy controls

To evaluate the potential of the differentially expressed miR-421 to diagnose early-stage gastric cancer patients, we further analyzed the expression levels of miR-421 in GC patients at an early stage (TNM stages I-II). The results showed that the expression levels of circulating miR-421 in healthy controls were significantly lower than those in early-stage gastric cancer patients 
$182(p<0.0001$, Fig.3A). The AUC values of miR-421 were $0.9907(95 \%$ confidence interval $(\mathrm{CI})=$ 1830.931 to 1.0 , sensitivity $=100 \%$ and specificity $=95.56 \%$, Fig. 3B). These results indicate that 184 miR-421 could be invoked as a novel biomarker for early diagnosis of gastric cancer.

185

\section{miR-421 had higher diagnostic value than traditional tumor markers for precancerous gastric lesions}

Based on the verified difference of miR-421 between the Pre and NC group (Fig. 1B,1C), ROC curves were constructed to further assess the role of miR-421 expression in the diagnosis of precancerous lesions. According to the different concentration of traditional tumor markers between the Pre group and the NC group (Figs. 4A,4B), their diagnostic values with miR421were further compared. ROC analyses showed that up-regulation of plasma miR-421 could discriminate Pre patients well from control subjects, with an AUC value of 0.873 (sensitivity= $66.29 \%$, specificity $=95.56 \%$, cutoff value: 3.23$)($ Fig.4C). Furthermore, as shown in table 4 and Figs. 4C \& 4D, the AUC of miR-421 was higher than CA153, CA211, and CA50, the sensitivity was higher than CA50, while the specificity and Youden index were higher than CA153, and CA50. Moreover, the FNR of miR-421 was lower than CA50, while the FPR was lower than CA153, and CA50. According to the comparison of the ROC curves, the diagnostic efficacy of miR-421 was considerably higher than CA153, CA211 and CA50 (all $p<0.05$, Table 5). These results indicate that miR-421 has high diagnostic value for precancerous patients.

\section{Up-regulation of miR-421 occurred initially in the precancerous diseases as well} as the early GC

We found that up-regulation of miR-421 not only distinguishes early-stage gastric cancer well, but can also well distinguish precancerous lesions from normal controls. However, it is not clear at what stage miR-421 begins to be up-regulated throughout the development of GC. Therefore, expressions of miR-421 in precancerous disease, and different clinical stages (I, II, III and IV) were further analyzed and compared. The results showed that the expressions of miR-421 were statistically different between the Pre cases and GC stages I, II, III and IV ( $p<0.05$, Fig.5). However, there was no difference of miR-421 expression between the GC stages II, III and IV and GC cases stage I. Moreover, compared to the expression in Pre cases, the biomarker was 
211 gradually up-regulated from the NC to the Pre and stage I cases, and finally, the maximum

212 expression value appeared in stage I cases (Table 6). The marked up-regulation of miR-421

213 occurred initially in precancerous disease as well as early-stage GC.

214 Association of miR-421 with clinicopathological features

215 In order to determine whether miR-421 levels in plasma were correlated with clinicopathological

216 features in patients with GC and precancerous lesions, the clinical data of 89 Pre and 90 GC-

217 proven cases were collected and analyzed. The high and low expression groups of each

218 parameter were calculated by the median of the relative quantity of miR-421. Correlation

219 analysis showed no statistically significant association of miR-421 expression with GC patients'

220 age $(p=0.0736)$, gender $(p=0.3265)$, TNM stage $(p=0.3531)$, differentiation degree $(p=$

$2210.5105)$, and histological type $(p=0.5079)$. Similarly, we found that there were no apparent

222 correlations of miR-421 expression with Pre patients' age $(p=0.5896)$, gender $(p=0.5934)$,

223 histological type $(p=0.6939)$, and Helicobacter pylori infection status $(p=0.3095)$ (Table 7).

\section{Discussion}

225 To date, there is no reliable non-invasive blood-based biomarker for the detection of

226

227

228

229

230

231

232

233

234

235

236

237

238

239

precancerous lesions and early-stage gastric cancer. We studied the diagnostic value of miR-421

in plasma in precancerous gastric lesions and GC patients. We found that the plasma expression levels of miR-421 were statistically significantly higher in early-stage GC and precancerous gastric lesions patients than in healthy controls. ROC analyses demonstrated that miR-421 has

high sensitivity and specificity in discriminating early-stage GC and precancerous gastric lesions patients from healthy controls. Our results indicated that miR-421 in plasma presented statistically significant diagnostic efficacy in detecting early GC as well as precancerous gastric lesions patients.

High-throughput analysis showed that miR-421 is localized on human chromosome1p34.23, and is one prominent member of the miR-200 family(Liu et al. 2015). Liu et al reported that miR-421 elevation in GC tissue(Liu et al. 2015; Zhao et al. 2015)and gastric juice(Zhang et al. 2012b)probably had implications in GC diagnosis. These results are consistent with our findings that miR-421 was highly expressed in the plasma of gastric cancer might have the potential 
240 diagnostic value in cancer. However, these researches are based on invasive, patients may not be

241 willing to accept, is bound to affect its promotion. Moreover, our study showed that miR-421

242 could achieve a satisfactory diagnostic efficiency in distinguishing GC patients from healthy

243 controls with an AUC of 0.981 (sensitivity $=96.67 \%$ and specificity $=95.56 \%$ ), higher than

244 miR-21, miR-20a and miR-378 tumor markers in previous studies (Liu et al. 2012; Mirzaei et al.

245 2016). In our study, we compared its diagnostic efficiency with that of traditional markers,

246 further clarifying its diagnostic value as a new tumor marker.

It is particularly important to improve the early diagnosis and prompt treatment of patients with gastric cancer. Researchers have illuminated the potential roles of several miRNAs in the early detection of cancers, such as lung carcinoma(Pan et al. 2018), colorectal neoplasm(Liu et al. 2018), and early-stage breast carcinoma(An et al. 2018). However, to date only a few miRNAs have been found effective as non-invasive biomarkers for the early detection of GC. Although the study such as the five-miRNA panel presented a high diagnostic value for the early stage of GC(Liu et al. 2012), the study did not include gastric precancerous lesions, and the subtle changes and performances of the miRNAs were not further elaborated. The miR-17-92 cluster was considered as a circulating biomarker for early detection of $\mathrm{GC}$, but there is no ROC data to support miRNA recognition of early stage cancer(Li et al. 2017). In our study, based on the detailed TNM stage characteristics of the GC sample, we explored the diagnostic value of plasma miR-421 in early gastric cancer. The results showed that the expression level of miR-421 could well distinguish early gastric cancer from normal controls, suggesting that miR-421 may have significant clinical value in the early diagnosis of gastric cancer.

Precancerous changes in the stomach can increase the risk of gastric cancer six-fold. Previous studies have reported that precancerous lesions are regulated by miRNAs(Li et al. 2014; Rotkrua et al. 2011).

Due to the absence of clinical manifestations of gastrointestinal metaplasia and atypical hyperplasia, there are few means for clinical non-invasive detection. Several circulating miRNAs, especially microRNA-196a (Chen et al. 2018), miR-16-5p, and miR-19b-3p(Zhang et 
and of GC progression. However, the comparative value of these miRNAs and traditional tumor markers in the diagnosis of gastric cancer and precancerous lesions has not been clarified, and an optimal circulating miRNA biomarker should have better specificity and sensitivity than traditional tumor markers. In the current study, we found that plasma miR-421 could well distinguish precancerous lesions of gastric cancer patients from healthy controls with an AUC of 0.872 (sensitivity $=66.29 \%$ and specificity $=95.56 \%$ ). Furthermore, the diagnostic efficacy of miR-421 was markedly higher than traditional tumor markers, such as CA153, CA211, and CA50.

The occurrence and development of gastric cancer is a multi-stage process, and miR-421 may be associated with a certain stage or the whole process. In this study, we further analyzed the trend of the up-regulation of miR-421 with the progression of GC. Results showed that the expression of plasma miR-421 in GC cases at stage I is no different from stages II, II, and IV, but plasma miR-421 in precancerous cases can be distinctly distinguished from GC at each stage, indicating that miR-421 occurred initially in precancerous lesions as well as early GC. Therefore, we speculate that the up-regulation in the miR-421 may be triggered by risk factors, especially chronic inflammation in the premalignant stomach, suggesting that a detailed investigation is needed.

\section{Conclusions}

Our findings demonstrate that plasma miR-421 may be a potential marker for precancerous lesions and early gastric cancer. This study provides us with a new insight into the role of miR421 in gastric cancer. These results could pave the way for the development of a novel biomarker for the early diagnosis of gastric cancer. Used this biomarker may allow a reliable indicator of gastric cancer at a much lower cost than methods currently available. The limitations of this study are that our normal control samples were relatively small, and most cancer samples were at an advanced stage. The exact use of miR-421 as an early plasma biomarker in clinical diagnosis of precancerous lesions and gastric cancer should be further explored in studies that use larger samples and in clinical trials. 


\section{REFERENCES}

An X, Quan H, Lv J, Meng L, Wang C, Yu Z, and Han J. 2018. Serum microRNA as potential biomarker to detect breast atypical hyperplasia and early-stage breast cancer. Future Oncol. 10.2217/fon-2018-0334

Chan AO, Wong BC, and Lam SK. 2001. Gastric cancer: past, present and future. Can J Gastroenterol 15:469-474.

Chen TH, Lee C, Chiu CT, Chu YY, Cheng HT, Hsu JT, Tsou YK, Wu RC, Chen TC, Chang NC, Yeh TS, and Lin KH. 2018. Circulating microRNA-196a is an early gastric cancer biomarker. Oncotarget 9:10317-10323.

Craanen ME, Dekker W, Ferwerda J, Blok P, and Tytgat GN. 1991. Early gastric cancer: a clinicopathologic study. $J$ Clin Gastroenterol 13:274-283.

He CZ, Zhang KH, Li Q, Liu XH, Hong Y, and Lv NH. 2013. Combined use of AFP, CEA, CA125 and CA19-9 improves the sensitivity for the diagnosis of gastric cancer. BMC Gastroenterol 13:87.

Hernando E. 2007. microRNAs and cancer: role in tumorigenesis, patient classification and therapy. Clin Transl Oncol 9:155-160.

Lee RC, and Ambros V. 2001. An extensive class of small RNAs in Caenorhabditis elegans. Science 294:862-864.

Li BS, Zhao YL, Guo G, Li W, Zhu ED, Luo X, Mao XH, Zou QM, Yu PW, Zuo QF, Li N, Tang B, Liu KY, and Xiao B. 2012. Plasma microRNAs, miR-223, miR-21 and miR-218, as novel potential biomarkers for gastric cancer detection. PLoS One 7:e41629. 10.1371/journal.pone.0041629

Li H, Wu Q, Li T, Liu C, Xue L, Ding J, Shi Y, and Fan D. 2017. The miR-17-92 cluster as a potential biomarker for the early diagnosis of gastric cancer: evidence and literature review. Oncotarget 8:45060-45071. 10.18632/oncotarget.15023

Li T, Lu YY, Zhao XD, Guo HQ, Liu CH, Li H, Zhou L, Han YN, Wu KC, Nie YZ, Shi YQ, and Fan DM. 2014. MicroRNA-296-5p increases proliferation in gastric cancer through repression of Caudal-related homeobox 1. Oncogene 33:783-793. 10.1038/onc.2012.637

Li Y, Cui X, Li Y, Zhang T, and Li S. 2018. Upregulated expression of miR-421 is associated with poor prognosis in non-small-cell lung cancer. Cancer Manag Res 10:2627-2633.

Liu H, Gao Y, Song D, Liu T, and Feng Y. 2015. Correlation between microRNA-421 expression level and prognosis of gastric cancer. Int J Clin Exp Pathol 8:15128-15132.

Liu H, Zhu L, Liu B, Yang L, Meng X, Zhang W, Ma Y, and Xiao H. 2012. Genome-wide microRNA profiles identify miR-378 as a serum biomarker for early detection of gastric cancer. Cancer Lett 316:196-203.

Liu X, Xu T, Hu X, Chen X, Zeng K, Sun L, and Wang S. 2018. Elevated circulating miR-182 acts as a diagnostic biomarker for early colorectal cancer. Cancer Manag Res 10:857-865. 10.2147/cmar.s158016

Lu J, Getz G, Miska EA, Alvarez-Saavedra E, Lamb J, Peck D, Sweet-Cordero A, Ebert BL, Mak RH, Ferrando AA, Downing JR, Jacks T, Horvitz HR, and Golub TR. 2005. MicroRNA expression profiles classify human cancers. Nature 435:834-838. 10.1038/nature03702

Mirzaei H, Khataminfar S, Mohammadparast S, Sales SS, Maftouh M, Mohammadi M, Simonian M, Parizadeh SM, Hassanian SM, and Avan A. 2016. Circulating microRNAs as Potential Diagnostic Biomarkers and Therapeutic Targets in Gastric Cancer: Current Status and Future Perspectives. Curr Med Chem 23:41354150.

Pan J, Zhou C, Zhao X, He J, Tian H, Shen W, Han Y, Chen J, Fang S, Meng X, Jin X, and Gong Z. 2018. A twomiRNA signature (miR-33a-5p and miR-128-3p) in whole blood as potential biomarker for early diagnosis of lung cancer. Sci Rep 8:16699. 
Pectasides D, Mylonakis A, Kostopoulou M, Papadopoulou M, Triantafillis D, Varthalitis J, Dimitriades M, and Athanassiou A. 1997. CEA, CA 19-9, and CA-50 in monitoring gastric carcinoma. Am J Clin Oncol 20:348353.

Rotkrua P, Akiyama Y, Hashimoto Y, Otsubo T, and Yuasa Y. 2011. MiR-9 downregulates CDX2 expression in gastric cancer cells. Int J Cancer 129:2611-2620. 10.1002/ijc.25923

Tsujiura M, Komatsu S, Ichikawa D, Shiozaki A, Konishi H, Takeshita H, Moriumura R, Nagata H, Kawaguchi T, Hirajima S, Arita T, Fujiwara H, Okamoto K, and Otsuji E. 2015. Circulating miR-18a in plasma contributes to cancer detection and monitoring in patients with gastric cancer. Gastric Cancer 18:271-279.

Wang Y, Liu Z, and Shen J. 2018. MicroRNA-421-targeted PDCD4 regulates breast cancer cell proliferation. Int J Mol Med.

Yang AP, Liu J, Lei HY, Zhang QW, Zhao L, and Yang GH. 2014. CA72-4 combined with CEA, CA125 and CA199 improves the sensitivity for the early diagnosis of gastric cancer. Clin Chim Acta 437:183-186. 10.1016/j.cca.2014.07.034

Yang R, Fu Y, Zeng Y, Xiang M, Yin Y, Li L, Xu H, Zhong J, and Zeng X. 2017. Serum miR-20a is a promising biomarker for gastric cancer. Biomed Rep 6:429-434.

Zhang J, Song Y, Zhang C, Zhi X, Fu H, Ma Y, Chen Y, Pan F, Wang K, Ni J, Jin W, He X, Su H, and Cui D. 2015. Circulating MiR-16-5p and MiR-19b-3p as Two Novel Potential Biomarkers to Indicate Progression of Gastric Cancer. Theranostics 5:733-745.

Zhang X, Cui L, Ye G, Zheng T, Song H, Xia T, Yu X, Xiao B, Le Y, and Guo J. 2012a. Gastric juice microRNA421 is a new biomarker for screening gastric cancer. Tumour Biol 33:2349-2355. 10.1007/s13277-012-0497$\mathrm{X}$

Zhang Y, Gong W, Dai S, Huang G, Shen X, Gao M, Xu Z, Zeng Y, and He F. 2012b. Downregulation of human farnesoid $\mathrm{X}$ receptor by miR-421 promotes proliferation and migration of hepatocellular carcinoma cells. Mol Cancer Res 10:516-522. 10.1158/1541-7786.mcr-11-0473

Zhao G, Xu L, Hui L, and Zhao J. 2015. Level of circulated microRNA-421 in gastric carcinoma and related mechanisms. Int J Clin Exp Pathol 8:14252-14256.

Zheng RS, Sun KX, Zhang SW, Zeng HM, Zou XN, Chen R, Gu XY, Wei WW, and He J. 2019. Report of cancer epidemiology in China, 2015. Zhonghua Zhong Liu Za Zhi 41:19-28.

Zhou S, Wang B, Hu J, Zhou Y, Jiang M, Wu M, Qin L, and Yang X. 2016. miR-421 is a diagnostic and prognostic marker in patients with osteosarcoma. Tumour Biol.

Zhou X, Ji G, Chen H, Jin W, Yin C, and Zhang G. 2015. Clinical role of circulating miR-223 as a novel biomarker in early diagnosis of cancer patients. Int J Clin Exp Med 8:16890-16898. 
Figure 1 (on next page)

MiR-421 expression level between different groups

MiR-421 expression level between different groups (A) Differential expression levels of reference miR-16( $\left.{ }^{*} p>0.05\right)$. (B) The $\Delta$ Ct values of miR-421 between different groups (****p $<0.0001)$. (C) The levels $\left(2^{-\Delta c t}\right)$ of miR-421 between different groups (****p $\left.<0.0001\right)$. The $\Delta \mathrm{Ct}$ values denote the normalized $\mathrm{Ct}$ value by subtracting the $\mathrm{Ct}$ value of miR-16 from that of miR-421. The lower $\Delta \mathrm{Ct}$ value means the higher level of miR-421 expression. The MannWhitney $\mathrm{U}$ test was used to determine statistical significance at the level of $p<0.05$. 

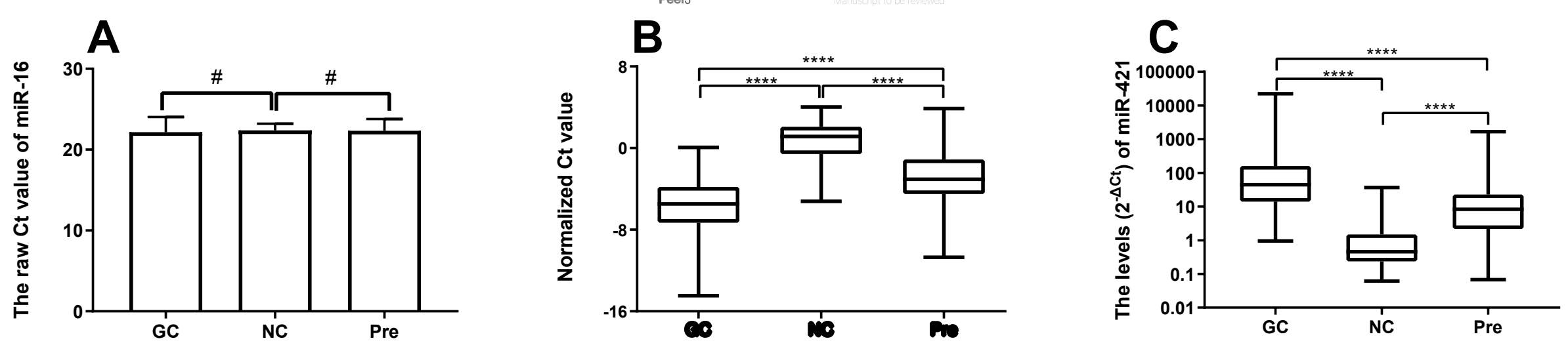
Figure 2

The ROC curves of miR-421 and traditional tumor markers for GC

(A) The AUC of miR-421was 0.981 ( $p<0.05)$. (B)The AUC of CEA, CA50, CA125, CA153 and CA211 were $0.672,0.754,0.645,0.656$ and 0.799 , respectively (all $p<0.05$ ).
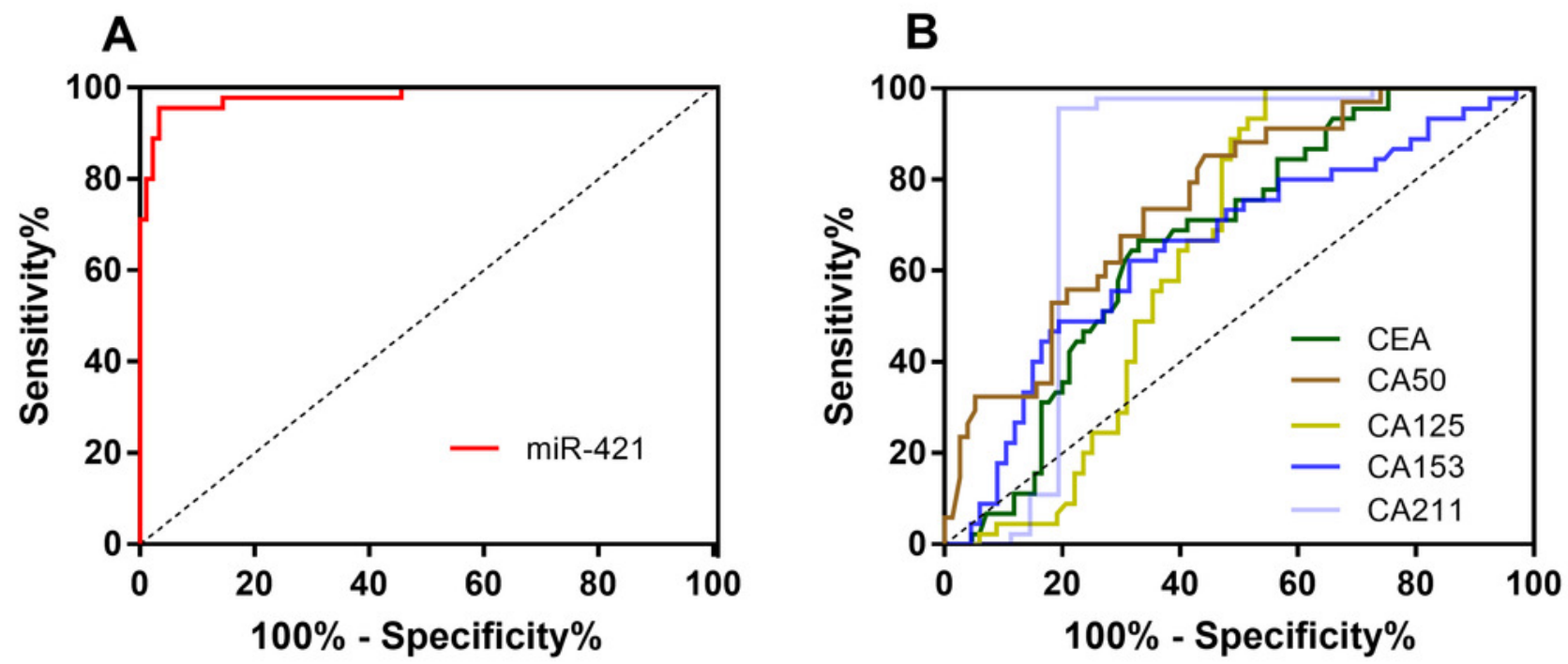
Figure 3

MiR-421 could distinguish early stage gastric cancer patients from healthy controls

(A) MiR-421 was up-regulated in early stages (TNM stage I-II) gastric cancer patients compared to healthy controls $(* * * * p<0.0001)$. (B) The ROC analysis for detection of earlystages gastric cancer patients from health controls using miR-421.

*Note: Auto Gamma Correction was used for the image. This only affects the reviewing manuscript. See original source image if needed for review.
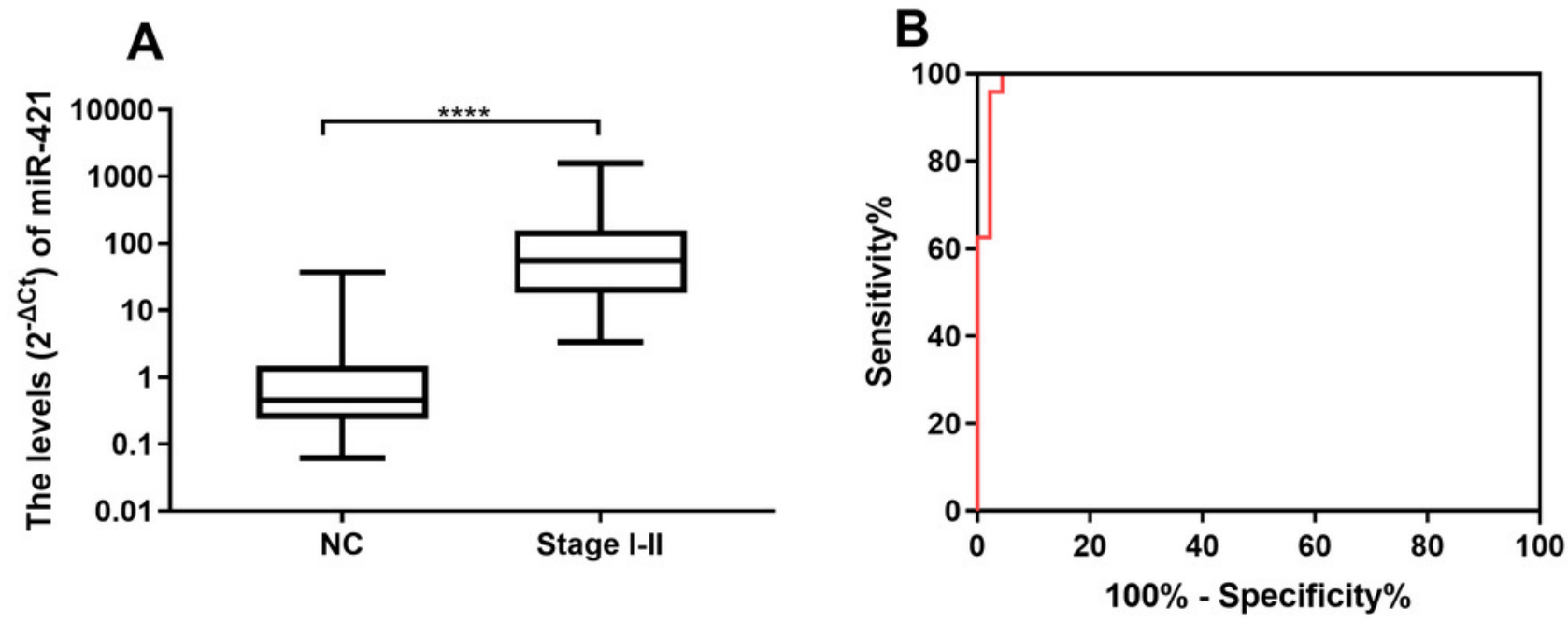
Figure 4

miR-421 had higher diagnostic value than traditional tumor markers for precancerous patients.

Differential expression levels of CA50(A) and CA211(B) between precancerous patients and healthy controls $(* p<0.01, * * * * p<0.0001)$. The ROC analysis for detection of precancerous using miR-421(C) and traditional tumor markers CA50 and CA211(D).

*Note: Auto Gamma Correction was used for the image. This only affects the reviewing manuscript. See original source image if needed for review.
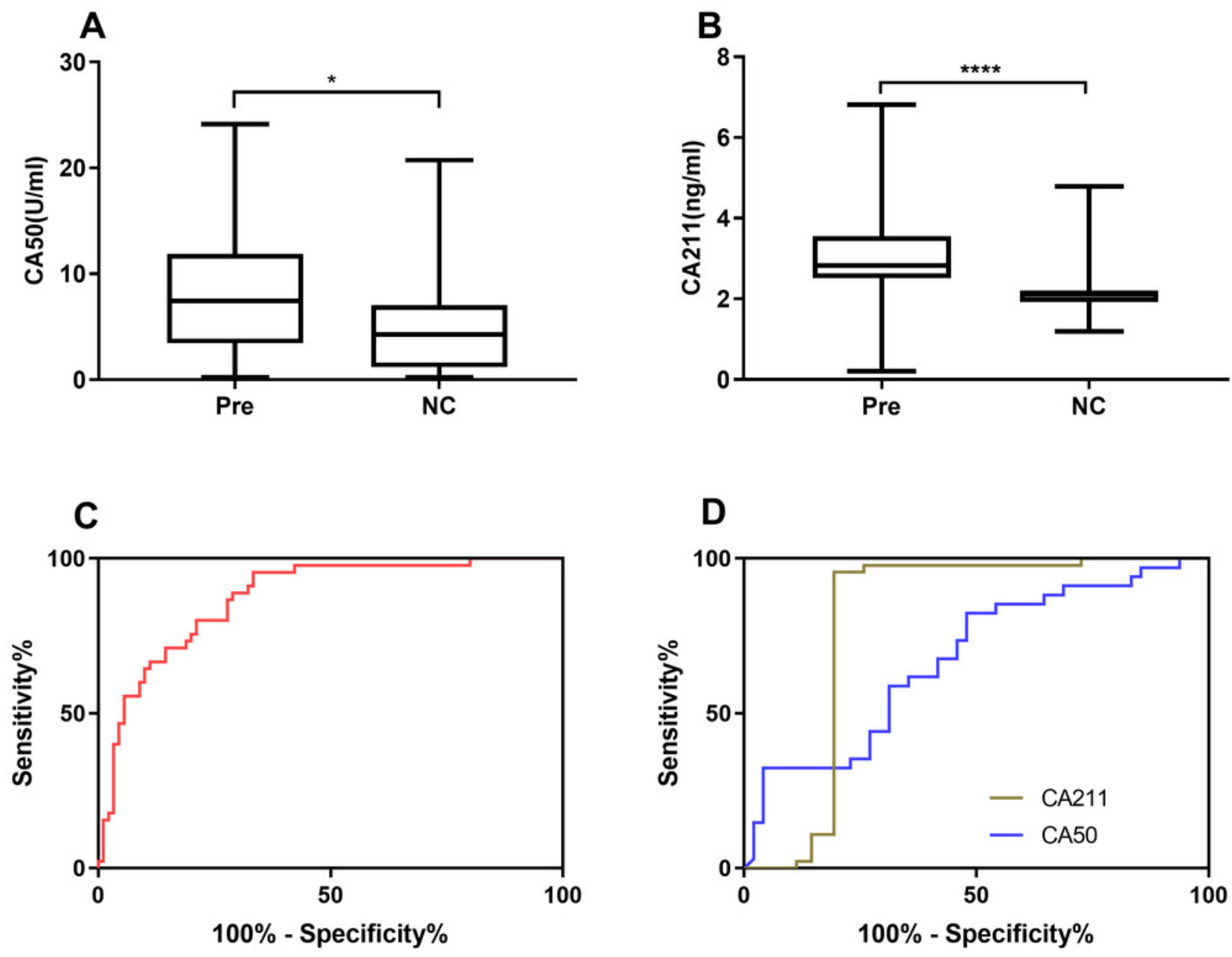
Figure $\mathbf{5}$ (on next page)

MiR-421 expression levels in plasma of GC cases with different TNM stages, PLGC cases and NC controls.

Mann-Whitney $\mathrm{U}$ test was used to determine statistical significance, $p<0.05$ was accepted as statistically significant. $* p<0.05, * * * * p<0.0001, \# p>0.05$. 


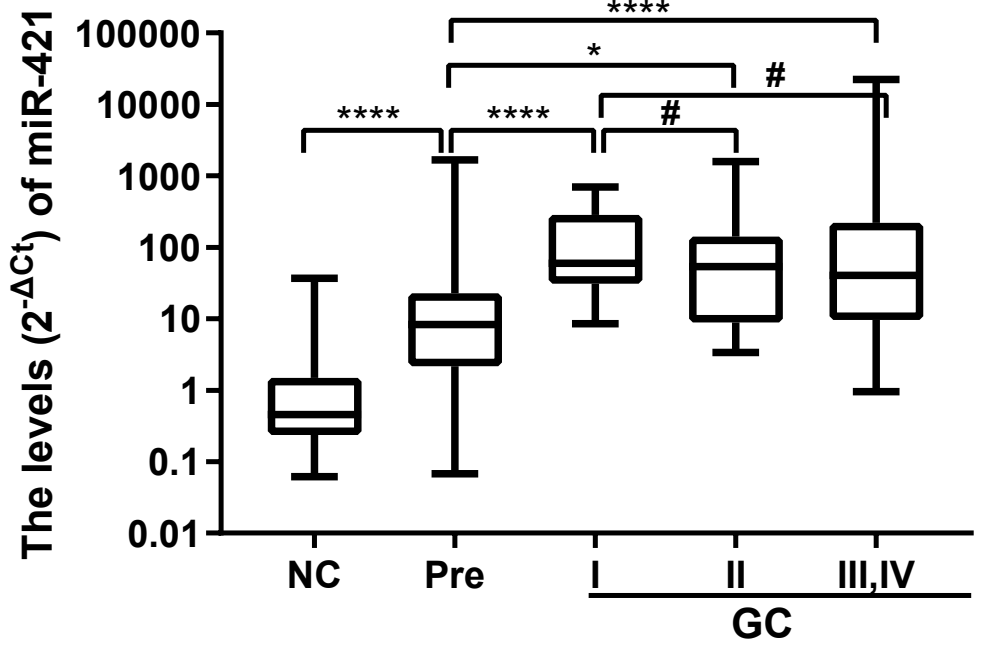




\section{Table 1 (on next page)}

Differential expression levels of traditional tumor markers in plasma of GC, Pre and health controls

${ }^{\mathrm{t}}$ For these categorical variables, the $p$ value were calculated by Student's t-test. MannWhitney $\mathrm{U}$ test was used to determine other categorical variables with statistical significance at the level of $p<0.05$. 
Table 1. Differential expression levels of traditional tumor markers in plasma of GC, Pre and health controls

\begin{tabular}{|c|c|c|c|c|c|c|c|c|}
\hline & $\mathbf{n}$ & $\begin{array}{c}\text { GC } \\
\text { mean } \pm \text { SD }\end{array}$ & $\mathbf{n}$ & $\begin{array}{c}\text { Pre } \\
\text { mean } \pm \text { SD }\end{array}$ & $\begin{array}{c}\text { Pre vs NC } \\
p \text { value }\end{array}$ & $\mathbf{n}$ & $\begin{array}{c}\mathrm{NC} \\
\text { mean } \pm \mathrm{SD}\end{array}$ & $\begin{array}{c}\text { GC vs NC } \\
p \text { value }\end{array}$ \\
\hline AFP & 85 & $(26.14 \pm 133.56)$ & 66 & $(3.32 \pm 1.72)$ & 0.0505 & 45 & $(3.69 \pm 1.25)$ & 0.3002 \\
\hline CEA & 85 & $(32.23 \pm 128.76)$ & 66 & $(1.82 \pm 1.39)$ & $0.3122^{\mathrm{t}}$ & 45 & $(2.08 \pm 1.20)$ & 0.0011 \\
\hline Ferritin & 80 & $(281.08 \pm 383.42)$ & 61 & $(270.11 \pm 302.39)$ & $0.1270^{t}$ & 45 & $(185.67 \pm 237.46)$ & 0.9908 \\
\hline CA125 & 68 & $(59.22 \pm 92.72)$ & 53 & $(12.50 \pm 7.78)$ & 0.9363 & 45 & $(11.07 \pm 4.05)$ & 0.0091 \\
\hline CA153 & 67 & $(13.44 \pm 17.70)$ & 50 & $(10.41 \pm 5.45)$ & $0.0065^{t}$ & 45 & $(13.67 \pm 5.83)$ & 0.0049 \\
\hline CA199 & 83 & $(107.03 \pm 251.90)$ & 57 & $(13.95 \pm 12.37)$ & 0.1071 & 45 & $(14.58 \pm 8.22)$ & 0.0705 \\
\hline CA211 & 62 & $(16.15 \pm 44.84)$ & 40 & $(2.96 \pm 1.40)$ & $<0.0001$ & 45 & $(2.09 \pm 0.48)$ & 0.0002 \\
\hline CA242 & 66 & $(19.17 \pm 39.24)$ & 45 & $(4.92 \pm 4.8)$ & $0.2976^{\mathrm{t}}$ & 36 & $(6.14 \pm 5.48)$ & 0.1425 \\
\hline CA50 & 77 & $(38.01 \pm 99.02)$ & 48 & $(8.18 \pm 5.97)$ & $0.0101^{\mathrm{t}}$ & 34 & $(4.91 \pm 4.46)$ & 0.0089 \\
\hline CA 724 & 75 & $(15.12 \pm 38.23)$ & 44 & $(2.49 \pm 2.87)$ & $0.2347^{\mathrm{t}}$ & 26 & $(3.32 \pm 2.59)$ & 0.2049 \\
\hline
\end{tabular}

${ }^{\mathrm{t}}$ For these categorical variables, the $p$ value were calculated by Student's t-test. 


\section{Table 2 (on next page)}

The diagnostic value of miR-421 and tumor markers for GC

AUC: Area under curve; FPR: False positive rate; FNR: False negative rate. Positive was defined as >cut-off value; Negative was defined as < cut-off value; The sensitivity, specificity, FPR, FNR, accuracy and Youden index were calculated based on the number of positive cases and negative cases. 
Table 2. The diagnostic value of miR-421 and tumor markers for GC.

\begin{tabular}{lccccccccc}
\hline & Cut-off & $\begin{array}{c}\text { Sensitivity } \\
\mathbf{( \% )}\end{array}$ & $\begin{array}{c}\text { Specificity } \\
\mathbf{( \% )}\end{array}$ & $\begin{array}{c}\text { FPR } \\
\mathbf{( \% )}\end{array}$ & $\begin{array}{c}\text { FNR } \\
\mathbf{( \% )}\end{array}$ & $\begin{array}{c}\text { Accuracy } \\
\mathbf{( \% )}\end{array}$ & $\begin{array}{c}\text { Youden } \\
\text { index (\%) }\end{array}$ & AUC & $p$ \\
\hline miR-421 & 3.23 & 96.67 & 95.56 & 4.44 & 3.33 & 96.30 & 92.22 & $0.981(0.942-0.997)$ & $<0.0001$ \\
CEA & 1.94 & 67.06 & 66.67 & 33.33 & 32.94 & 66.93 & 33.73 & $0.672(0.584-0.752)$ & 0.0002 \\
CA125 & 18.75 & 45.59 & 100.00 & 0 & 54.41 & 67.26 & 45.59 & $0.645(0.549-0.732)$ & 0.0055 \\
CA153 & 11.31 & 68.66 & 62.22 & 37.78 & 31.34 & 66.07 & 30.88 & $0.656(0.561-0.743)$ & 0.0034 \\
CA211 & 2.2 & 80.65 & 95.65 & 4.35 & 19.35 & 86.96 & 76.30 & $0.799(0.711-0.871)$ & $<0.0001$ \\
CA50 & 7.96 & 55.84 & 85.29 & 14.71 & 44.16 & 64.86 & 41.14 & $0.754(0.663-0.831)$ & $<0.0001$ \\
\hline
\end{tabular}

AUC: Area under curve; FPR: False positive rate; FNR: False negative rate. Positive was defined as $>$ cut-off value; Negative was defined as < cut-off value; The sensitivity, specificity, FPR, FNR, accuracy and Youden index were calculated based on the number of positive cases and negative cases. 


\section{Table 3(on next page)}

The comparison of ROC curves between miR-421 and tumor markers

ROC curves comparisons were analyzed by using MedCalc statistical software with statistical significance at the level of $p<0.05$. 
1 Table 3. The comparison of ROC curves between miR-421 and tumor markers

\begin{tabular}{lcccccccccc}
\hline \multirow{2}{*}{$\begin{array}{l}\text { ROC curves } \\
\text { comparison }\end{array}$} & \multicolumn{2}{c}{ CEA } & \multicolumn{2}{c}{ CA125 } & \multicolumn{2}{c}{ CA153 } & \multicolumn{2}{c}{ CA211 } & \multicolumn{2}{c}{ CA50 } \\
& 2.418 & 0.0156 & 1.896 & 0.0580 & 2.021 & 0.0432 & 3.975 & 0.0001 & 3.343 & 0.0008 \\
\hline
\end{tabular}

2 ROC curves comparisons were analyzed by using MedCalc statistical software with statistical significance at

3 the level of $p<0.05$. 


\section{Table 4 (on next page)}

The diagnostic value of miR-421 and tumor markers for Pre.

The sensitivity, specificity, FPR, FNR, accuracy and Youden index were calculated based on the number of positive cases and negative cases. AUC, FPR and FNR, Positive and Negative as stated in Table 2. 
Table 4. The diagnostic value of miR-421 and tumor markers for Pre.

\begin{tabular}{lccccccccc}
\hline & Cut-off & $\begin{array}{c}\text { Sensitivity } \\
(\mathbf{\%})\end{array}$ & $\begin{array}{c}\text { Specificity } \\
\mathbf{( \% )}\end{array}$ & $\begin{array}{c}\text { FPR } \\
\mathbf{( \% )}\end{array}$ & $\begin{array}{c}\text { FNR } \\
\mathbf{( \% )}\end{array}$ & $\begin{array}{c}\text { Accuracy } \\
\mathbf{( \% )}\end{array}$ & $\begin{array}{c}\text { Youden } \\
\text { Index (\%) }\end{array}$ & AUC & $p$ \\
\hline miR-421 & 3.23 & 66.29 & 95.56 & 4.44 & 33.71 & 76.37 & 61.85 & $0.872(0.804-0.924)$ & $<0.0001$ \\
CA153 & 11.81 & 72.00 & 60.00 & 40.00 & 28.00 & 66.32 & 32.00 & $0.679(0.576-0.772)$ & 0.0012 \\
CA211 & 2.2 & 85.00 & 95.56 & 4.44 & 15.00 & 90.56 & 80.56 & $0.844(0.750-0.914)$ & $<0.0001$ \\
CA50 & 7.26 & 52.08 & 82.35 & 17.65 & 47.92 & 64.63 & 34.44 & $0.677(0.564-0.776)$ & 0.0034 \\
\hline
\end{tabular}

The sensitivity, specificity, FPR, FNR, accuracy and Youden index were calculated based on the number of positive cases and negative cases. AUC, FPR and FNR, Positive and Negative as stated in Table 2. 


\section{Table 5 (on next page)}

The comparison of ROC curves among miR-421 and tumor markers for Pre

ROC curves comparisons were analyzed by using MedCalc statistical software with statistical significance at the level of $p<0.05$. 
1

2

3

4

(1)

Table 5. The comparison of ROC curves among miR-421 and tumor markers for Pre

\begin{tabular}{lccccccc}
\hline ROC curves & \multicolumn{2}{c}{ CA153 } & \multicolumn{2}{c}{ CA211 } & \multicolumn{2}{c}{ CA50 } \\
comparison & $\mathrm{Z}$ & $p$ & $\mathrm{Z}$ & $p$ & $\mathrm{Z}$ & $p$ \\
\hline miR-421 & 2.211 & 0.027 & 4.414 & $<0.0001$ & 1.997 & 0.0458 \\
\hline
\end{tabular}

ROC curves comparisons were analyzed by using MedCalc statistical software with statistical significance at the level of $p<0.05$. 


\section{Table 6(on next page)}

The levels $\left(2^{-\Delta c t}\right)$ of miR-421 between different groups

${ }^{\text {a }} \boldsymbol{p}$ represents the comparison of Pre between GC with I,II,IllandVI, respectively. ${ }^{b} \boldsymbol{p}$ represents the comparison of I between GC withll, IllandVI, respectively 
Table 6 The levels (2- $\Delta \mathrm{Ct})$ of miR-421 between different groups

2

${ }^{\text {a } P}$ represents the comparison of Pre between GC with I, II, IIIandVI, respectively.

$11{ }^{\mathbf{b}} \boldsymbol{P}$ represents the comparison of I between GC with II, IIIandVI, respectively 


\section{Table 7 (on next page)}

The relationships between the expression levels of miR-421(2- $\left.2^{-\Delta \mathrm{t}}\right)$ in plasma and clinicopathological factors of patients with precancerous gastric lesions and gastric cancer 


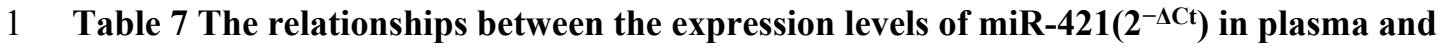

2 clinicopathological factors of patients with precancerous gastric lesions and gastric cancer

3

4

\begin{tabular}{|c|c|c|c|c|c|}
\hline \multirow[t]{2}{*}{ Characteristics } & \multirow[t]{2}{*}{ Case No. } & \multicolumn{2}{|c|}{$\begin{array}{l}\text { miR-421 } \\
\text { level }\left(2^{-\Delta C t}\right)\end{array}$} & \multirow[t]{2}{*}{$\chi 2$ test } & \multirow{2}{*}{$\begin{array}{l}P \\
\text { value }\end{array}$} \\
\hline & & High & Low & & \\
\hline GC Total cases & 90 & 45 & 45 & & \\
\hline \multicolumn{6}{|l|}{ Age(years) } \\
\hline$<60$ & 30 & 11 & 19 & 3.2 & 0.0736 \\
\hline$\geq 60$ & 60 & 34 & 26 & & \\
\hline \multicolumn{6}{|l|}{ Gender } \\
\hline Male & 68 & 32 & 36 & 0.9626 & 0.3265 \\
\hline Female & 22 & 13 & 9 & & \\
\hline \multicolumn{6}{|l|}{ TNM stage } \\
\hline $\mathrm{I}$ & 16 & 11 & 5 & 3.261 & 0.3531 \\
\hline II & 9 & 5 & 4 & & \\
\hline III & 23 & 12 & 11 & & \\
\hline VI & 40 & 17 & 23 & & \\
\hline \multicolumn{6}{|l|}{ Diferentiation degree } \\
\hline High and moderate & 25 & 11 & 14 & 0.433 & 0.5105 \\
\hline Poor & 46 & 24 & 22 & & \\
\hline \multicolumn{6}{|l|}{ Histological type } \\
\hline Adenocarcinoma (A) & 65 & 31 & 34 & 2.324 & 0.5079 \\
\hline Mucinous carcinoma(M) & 2 & 0 & 2 & & \\
\hline Signet ring cell carcinoma(S) & 5 & 3 & 2 & & \\
\hline A with S & 11 & 6 & 5 & & \\
\hline Pre Total cases & 89 & 45 & 44 & & \\
\hline \multicolumn{6}{|l|}{ Age(years) } \\
\hline$<60$ & 41 & 22 & 19 & 0.2916 & 0.5892 \\
\hline$\geq 60$ & 48 & 23 & 25 & & \\
\hline \multicolumn{6}{|l|}{ Gender } \\
\hline Male & 46 & 22 & 24 & 0.2851 & 0.5934 \\
\hline Female & 43 & 23 & 20 & & \\
\hline \multicolumn{6}{|l|}{ Histological type } \\
\hline Intestinal metaplasia & 82 & 41 & 41 & 0.1316 & 0.6939 \\
\hline Atypical hyperplasia and other type & 7 & 4 & 3 & & \\
\hline \multicolumn{6}{|l|}{ HP infection status } \\
\hline HP (-) & 38 & 20 & 18 & 1.033 & 0.3095 \\
\hline $\mathrm{HP}(+)$ & 16 & 6 & 10 & & \\
\hline
\end{tabular}

